\title{
Automatic License Plate Recognition from Live streaming using Stroke width Transform and Artificial Neural Network
}

\author{
Dr. Heena Kher ${ }^{\text {* }}$, Dr. Hetal Patel ${ }^{2}$ and Tejendra Panchal ${ }^{3}$ \\ ${ }^{1 *}$ Assistant Professor, Electronics and Communication Engineering Department, ADIT, New V. V. Nagar, Gujarat, India \\ ${ }^{2}$ Professor and Head, Electronics and Communication Engineering Department, ADIT, New V. V. Nagar, Gujarat, India \\ ${ }^{3}$ PG Student, Electronics and Communication Engineering Department, ADIT, New V. V. Nagar, Gujarat, India
}

\begin{abstract}
As the public and private transportation system increases, there is an urgent need of an Automatic License Plate Recognition (ALPR) system. ALPR system is equipped with many intelligent surveillance systems like road traffic management, security management and automatic toll collection system, etc. The basic steps in ALPR were accurate localization of number plate and recognition of license plate characters, which bears on the overall system accuracy. A license plate detection method was produced to find number plates from a live snap shots of a video stream showing the movement of all the vehicles in various conditions such as, non-uniform illumination, vehicle speed, background and foreground color, different weather condition, occlusion within image, etc. In this composition, the detection of a license plate from an image, stroke width transform was applied and been simulated on live snapshots. The artificial neural network based character and number recognition was applied on the detected license plate. The proposed algorithm gives $98 \%$ of license plate detection accuracy and $92.7 \%$ of number plate recognition accuracy.
\end{abstract}

Keywords- Automatic Licence Plate Recognition (ALPR), Stroke Width Transform (SWT), Artificial Neural Network (ANN)

\section{Introduction}

This paper addresses the issue of Automatic License Plate Recognition (ALPR) system. The raising increment of contemporary urban and National Street arranges throughout the most recent three decades arose the need of proficient observing and administration of road traffic.

License plate is a unique identifier of any vehicle and it is attached to vehicle for official identification purpose. Recent license plates in India have many high security features added in that. Many techniques are investigated by different researchers on license plate segmentation and registration. LPR is a challenging area of research because of its variety of applications. It can be used for automatic toll collection, in the parking lot for automatic ticketing, for vehicle tracking during violation of traffic signal and for security control.

For License Plate (LP) detection, Stroke Width Transform (SWT) was used, Connected Components were used for Segmentation and Artificial Neural Network (ANN) was used for LP Recognition. under which the subject for recognition needs to place. Usually ANN is widely used in recognition purpose where difficulties/ complexity need to be less. Many ANN algorithms are available but for the particular application, one has to make a decision based on the outcomes best suit for itself. Training can be done by providing the feature vector to the network neurons with number of fields in which one have to distinguish the data. Number of input neurons is same as the features extracted and the output neurons are the number of fields in which ANN will classify the data. Middle layer can have a number of neurons higher or lesser then the input layers.

One has to make the choice on number of neurons for middle layer based on the outcomes. Middle layer can't have the neurons more than the double of input layer neurons. If the number of features are extracted not enough to classify the data set then one have to make a wise decision on selecting the feature set. Middle layer usually have slop on training function where as output layer with the linear training function gives better output as it truncate output to specific range.

ANN is used to find out the best probable region/group

*Corresponding Author: e-mail: heena.adit@gmail.com,

Tel-+91-9909018627

ISSN 2320-7590

(C) 2018 Darshan Institute of Engg. \& Tech., All rights reserved 


\section{Related Work}

There are several methods used for license plate detection such as edge extraction, morphological operations, saliency features, Hough transform, Histogram analysis, fuzzy based or neural network based for color or grayscale classification. Edge extraction methods are faster than other methods but it is very sensitive to unwanted edges which are generated from the region around licenses plate. Hough transform method is used for line detection, so it is applicable where the image is having large plate area where we can get the shape of license plate defined by lines. But still it requires large memory space for the coordinates and also requires high computation time. Histogram analysis cannot be applicable where the license plate is having tilt or pan. Morphological operations are not applicable where image is noisy and will require large time. License plate localization is very important step which provides only LP region from the input image.

Feature extraction for an offline license plate recognition system based on global features is suggested by Mohindra and Shukla [1]. They have tested their algorithm on 100 real time images captured at different times in a day. They have used edge detection method, connected component analysis and discrete radon transform method for image segmentation. The efficiency of recognizing each character is $95 \%$ and total time taken for one image recognition is 15 seconds.

Edge finding method and window filtering is suggested by Kranthi et al. [2]. Edge finding method is not giving significant output when image is having complex background because it is very sensitive to unwanted edges. So windowing method where intensity summation in horizontal and vertical direction is calculated that will provide proper detection of license plate. For object enhancement they have used scale down, sorting of intensity pixel and then multiplication of top $20 \%$ pixel by 2.55 , which provide better result compared to histogram equalization technique.

Abbas et al. [3] suggested vertical edge based car license plate detection method. For low resolution image of size $352 *$ 288 , in which they have used adaptive thresholding technique followed by vertical edge detection using $2 * 4$ mask and proved that its performance is faster $(47.7 \mathrm{~ms})$ than the sobel and getting $91.4 \%$ correct detection. Advantages are like able to process low resolution image and with complex background, tolerance to lighting, tilt, varied sizes and designs of LPs. But their algorithm is highly complex.

Adaptive thresholding using the integral image described by Bradley and Roth [4], this type of thresholding is suitable in spatial variations in illumination. Integral image is summation of corresponding row and column and then different local threshold is calculated based on neighboring pixel values. It is highly robust when illumination changes occur in the image.
Nipa et al. [6] proposed a case where LP's are captured at an angle, in that skew correction is required and Harris corner detection is used to find features of license plate. For Harris corner detection thresholding, gradient, Gaussian smoothing and non maxima suppression is applied. Principle component analysis is used to extract only the meaningful data. It consists of mean, covariance matrix, Eigen value and Eigen vector calculation, finding maximum Eigen value and then corresponding Eigen vector is to be determined. Their algorithm works well for both positive and negative skewed images with less computation complexity. But the distance between camera and license plate image is very less.

Intrinsic rules saliency is used for automatic license plate localization by Paunwala and Patnaik [8]. Firstly, candidate region identification using adaptive edge density and connected component analysis is done, then three functions are determined, one is density variance measurement for uniformity check, second is scan line variance for measurement of transition variance and third is edge angle analysis for identification of skewed text region. From these three functions, final $\mathrm{F}$ is calculated and if value of $\mathrm{F}$ is higher than some predefined threshold then it is considered as LP otherwise it is discarded. They got robust result on a mixed data set with high diversity. This method has advantages like detection box have high precision and it is independent of LP style, scaling, rotation and partial occlusion.

Epshtein et al. [11] discovered new algorithm for text detection using stroke width transform, in which edge detection, stroke width calculation, finding letter region, filtering and combining words are the steps followed for text detection in natural image. This algorithm is suitable for handwritten text detection, for the text varying in size, different language text and for the text having different orientation. Limitation of this algorithm is when the letters are very small and close to each other then in will be group together in stroke width labeling phase.

To detect text, Jin Lee [12] proposed an effective machine learning based algorithm for classifying two or more classes known as Adaboost. This algorithm constructs a strong classifier from a combination of weak classifiers. They have used 6 types of feature sets that are variance and expectation of XY derivatives, local energy of Gabor filter, statistical texture measure of image histogram, measurement of variance of wavelet coefficient, edge detection and edge interval calculation and connected component analysis. There are several boosting algorithms which will improve performance of Adaboost like real Adaboost, Gentle Adaboost and Modest Adaboost and performance of these methods are compared in terms of precision, recall and f-measure. Precision is the performance metrics - the fraction of text window which are correctly classified as text. Recall - the fraction of all text windows which are correctly identified and $\mathrm{f}-$ measure is the 
harmonic mean of the precision and recall. For best performance, precision, recall and f-measure should be one.

License Plate detection algorithm using statistical features and LP templates are proposed by Kim et al. [13]. First, the entire image is searched and candidate areas based on the gradient features are located, the statistical features are calculated for region of interest (ROI), the candidates are examined to verify whether it contains LP and then LP templates are matched with ROI. Limitation of this algorithm is that it is difficult to construct general LP templates and it can work on a fixed scale.

Naito et al. [14] suggested Japanese license plate recognition method for passing vehicles under outside environment. They have used adaptive binarization, extraction of character region, hypothesis generation of registration numbers and then template matching for each hypothesis. Their algorithm is very efficient and able to process rotated and low contrast images. But the limitation of this method is license plate size should be fixed.

Text detection using Stroke Width Transform (SWT) has been suggested by Epshtein et al. [11]. SWT is a novel image operator to find out stroke width for each image pixel. The work presented in this paper gives good results for text detection in natural images. The speed of execution is also fast than the previously published techniques. The method is robust to a wide range of imaging conditions, such as color noise, blur, occlusions, etc.

Kocer and Cevik [22] proposed Artificial Neural Networks (ANN) based vehicle license plate recognition. They have used canny operator and edge strength for localization of license plate. Contrast extension, median filtering and blob coloring method have been used for segmentation of characters and finally feature extraction and classification is done by using ANN.

\section{Proposed System}

A license plate is the special characteristic for distinguishing number of vehicles. The fundamental issues progressively license plate recognition are the exactness and the recognition speed.

Nature of calculations utilized as a part of a license plate finder decides the pace and precision of the license plate identification. Figure 1 shows block diagram of proposed system which includes Capture and LP detection, Segmentation of Characters and Recognition.
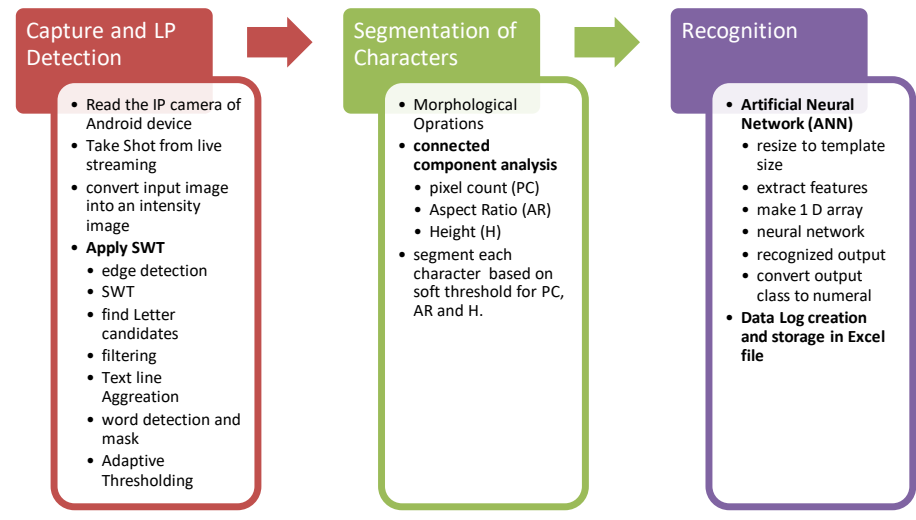

Figure 1. Block diagram of Proposed System

Real-time implementation of an Algorithm to evaluate the performance of an algorithm in real time without storing data on local drive, live streaming of a video data from an IP camera needs to be accessed. Once we get image, SWT is applied.

SWT is a novel image operator that will find a stroke width for each pixel. This operator is local and data dependent which makes it fast and robust. It is able to detect text regardless of its scale, direction, font and language.

\subsection{Stroke Width Transform}

3.1.1 Stroke Width: The SWT is a local operator which computes width of the stroke. The output of the SWT is an image of size same as input image where each element contains the width of the stroke associated with the pixel.

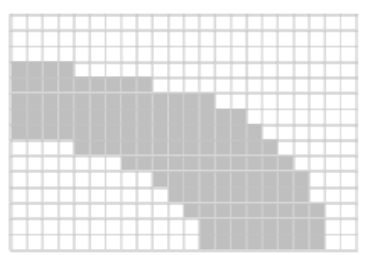

(a)

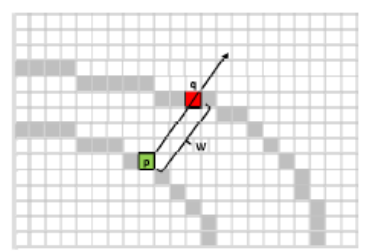

(b)

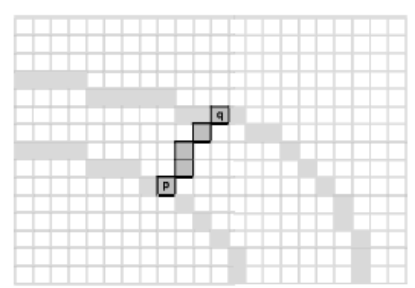

(c)

Figure 2. (a) A typical Stroke (b) Canny edge detection (c) Stroke width (courtesy: Epshtein et al. [11])

Figure 2 shows a typical stroke. The pixels of the stroke in this are darker than the background pixels. After edge 
detection, for any ' $p$ ' pixel on the boundary of the stroke, searching in the direction of the gradient at ' $p$ ', directs to find ' $q$ ' which is the corresponding pixel on the other side of the stroke and storing the width. Each pixel will assign by the minimum width of the stroke.

The image gradient is a directional change of intensity in an image. It is a measure of image changes and provides magnitude and direction.

For a image $f(x, y)$ the gradient of $f$ at coordinates $(x, y)$ is defined as the two dimensional column vector as mentioned in equation 1.

$$
\nabla f=\operatorname{grad}(f)=\left[\begin{array}{l}
g_{x} \\
g_{y}
\end{array}\right]=\left[\begin{array}{l}
\frac{\partial f}{\partial x} \\
\frac{\partial f}{\partial y}
\end{array}\right]
$$

Magnitude $M(x, y)$ and angle $\theta(x, y)$ is described by equation 2 and 3 respectively. Magnitude tells us how quickly image is changing while direction tells us the direction in which the image is changing most rapidly.

$$
\begin{aligned}
& M(x, y)=\operatorname{mag}(\nabla f)=\sqrt{g_{x}^{2}+g_{y}^{2}} \\
& \theta(x, y)=\tan ^{-1} \frac{g_{y}}{g_{x}}
\end{aligned}
$$

The initial value of each element of the SWT is set to $\infty$. First compute edges and then find out gradient direction of each pixel ' $p$ ' as shown in fig. 3(b). If ' $p$ ' lies on a stroke boundary then traverse in the gradient direction until another edge pixel $q$ is found.

3.1.2 Finding Letter Candidates: The output of the previous step is an image where each pixel contains the width of the most likely stroke. The next step is to identify letter candidates from the stroke width image. Two neighboring pixels may be grouped together it they have similar stroke width. Connected component labeling can be used for binary image but here input is not binary so modified method should be used. Group two neighboring pixels if their SWT ratio is lower than 3. This rule guarantees that strokes with smoothly varying widths can be grouped together.

3.1.3 Word Detection: The next step is to group letters. Generally, license plate consists of 8 to 10 characters which can be grouped together and it is very significant filtering mechanism which will remove randomly scattered noise. For this step, height ratio, the distance between letters and average colors parameters are optimized based on the performance of training data set.
3.1.4: Adaptive Thresholding: Adaptive thresholding is applied to achieve better thresholding other than the traditional Otsu method. A local window of size $10 \times 10$ is used for thresholding. Local mean within the window is utilized to set the threshold at particular instance. Adaptive threshold is one type of soft threshold so that the result of different input data doesn't affect much on output side.

Adaptive thresholding result can have some extra unnecessary noise parts / spurs. Unwanted parts need to be removed for smooth and fast processing when forwarded for the segmentation stage.

3.2 Connected Component Analysis : An integrated approach based on connected components region properties was applied for segmentation of LP characters. Pixel labeling, Aspect ratio (AR), Pixel Count (PC), area and height parameters for each connected component was used.

3.2.1: Labeling : Labeling of each connected pixel was done based on 8 - connectivity. Each different part was labeled differently.

3.2.2: Aspect Ratio (AR): It is one of the controlling parameter to distinguish LP character from noise/ non character region. Region which not falls under AR range was not used for further processing.

3.2.3: Ratio of Pixel Count (PC) / Area: The parameter Area of a labeled region was computed based on its bounding box parameters. The PC was computed for each labeled region. For each region the ratio of PC by area was used for selection of LP character. Upper and lower bound of area was decided based on the properties extracted from CC.

3.2.4: Height $(H)$ : It's easier to eliminate the noisy/ non character region by simply considering the height parameter and height greater than the soft threshold will be eliminated.

3.3 Character Recognition: Artificial Neural Network (ANN) had been used to recognize the segmented characters. Levenberg-Marquardt algorithm (LMA) had been used as it gives the best match for weighting the neurons during the training period.

The steps needed for segmented character recognition are as follows.

1. Create the data set which needs to be classified: numeral as well as characters have been generated of particular size (42 X 24) and stored locally on computer. For better recognition, distorted and/or a bit noisy extra characters or numbers are also added.

2. Extract the features: Features are useful parameters to distinguish desired object from others. Features like area, perimeter, Euler number, eccentricity, 
orientation, length of line, cross section etc. had been used.

3. Train the ANN for all the features extracted from the data set. Total 242 input data set images were used.

4. Network is checked against the testing data base. Total 38 images were used for testing purpose. Result of testing with the ANN network into the notepad for instant reference and into the excel file for creation of $\log$.

\section{Results and Discussion}

The ALPR algorithm for live snapshots of stream video file was implemented on a system with 64 bit OS, x64 based processor @ $1.80 \mathrm{G} \mathrm{Hz}$, RAM 4 GB using MATLAB. The RGB image captured through Android phone Samsung Note 3 with pixel resolution $1080 \times 1920$ was used as an input image. The Android app IP Webcam was used for capturing the snaps from live streaming. The Android phone used as a camera for ALPR system was mounted on entrance of college premises. Fig. 3, 4, 5, 6, 7, 8 and 9 shows the process flow of the proposed algorithm. Fig. 11shows the notepad output of the input tested image which gives exact recognition.

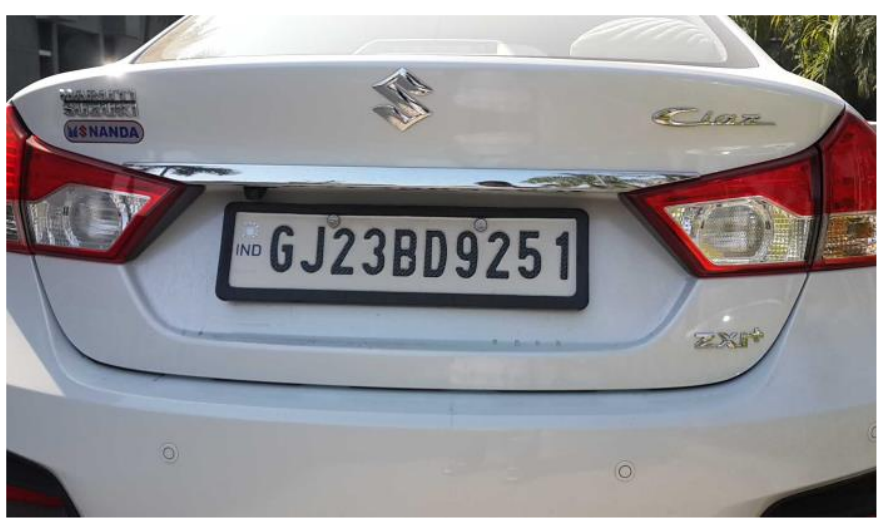

Figure 3. Input Image

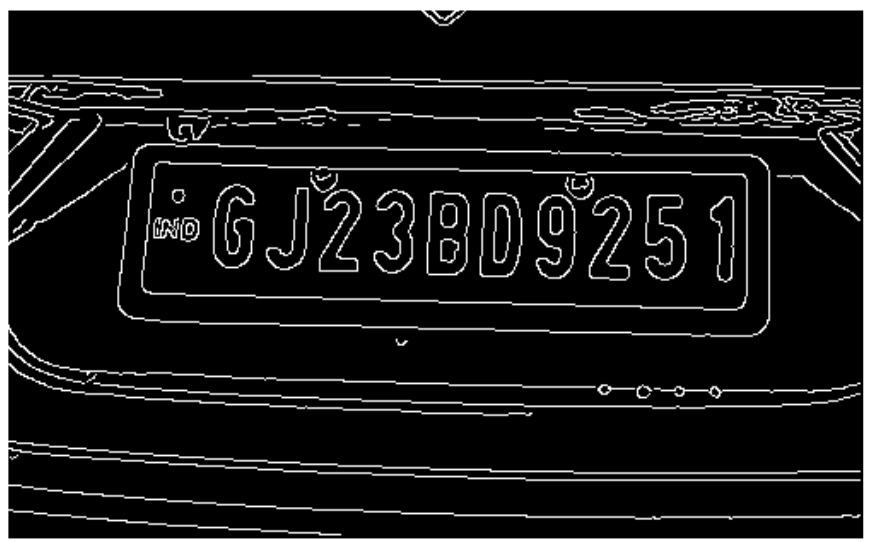

Figure 4. Canny Edge Detection

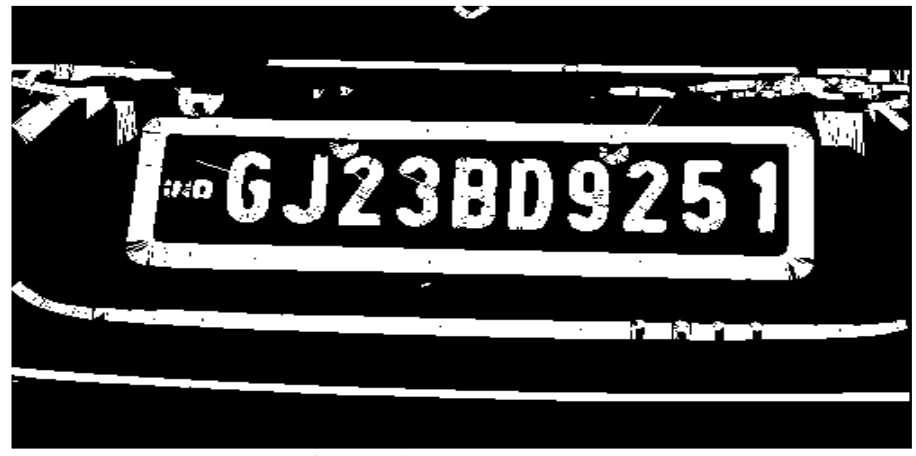

Figure 5. Stroke Width

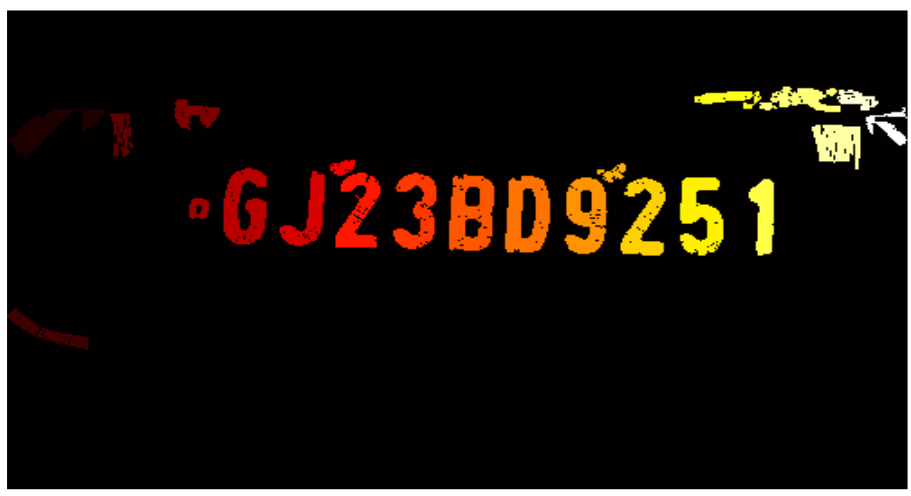

Figure 6. Labeling and Letter Candidate

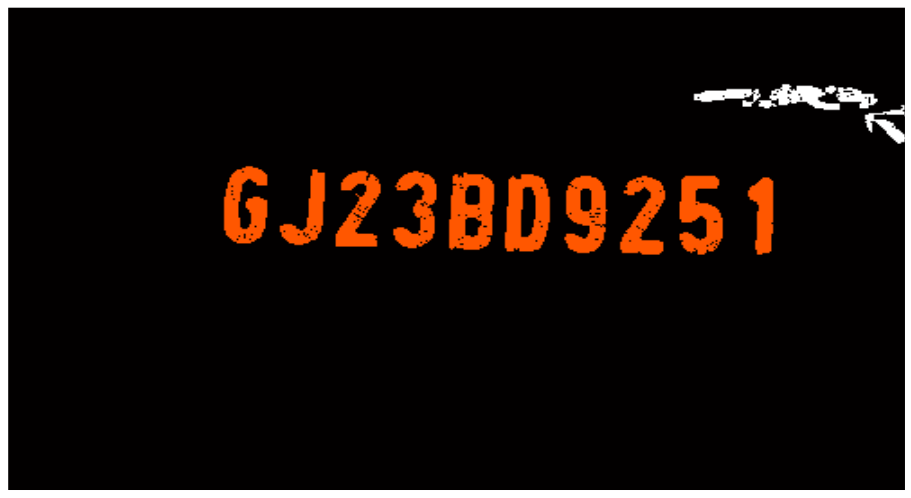

Figure 7. License Plate Detection

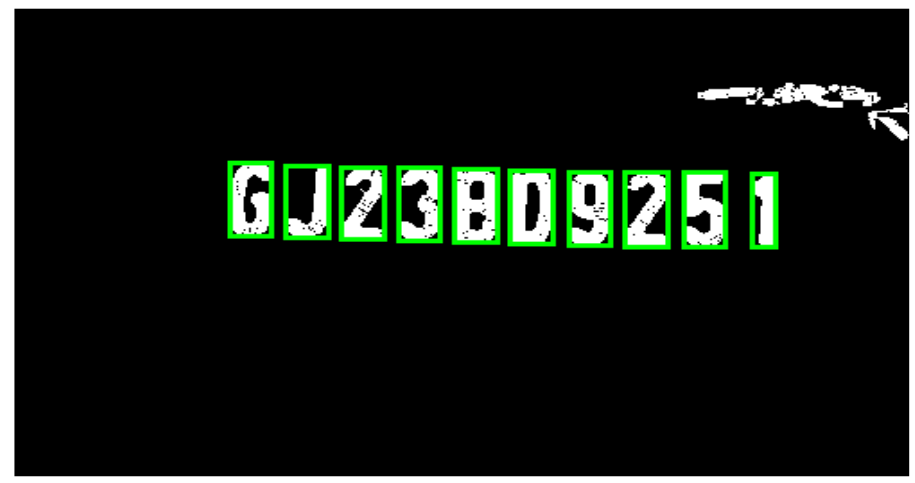

Figure 8. License Plate Segmentation 


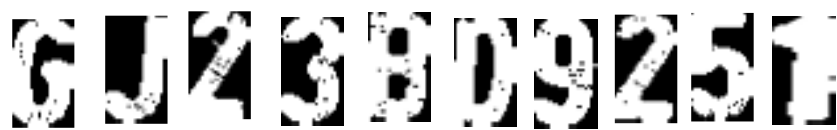

Figure 9. Segmented Characters

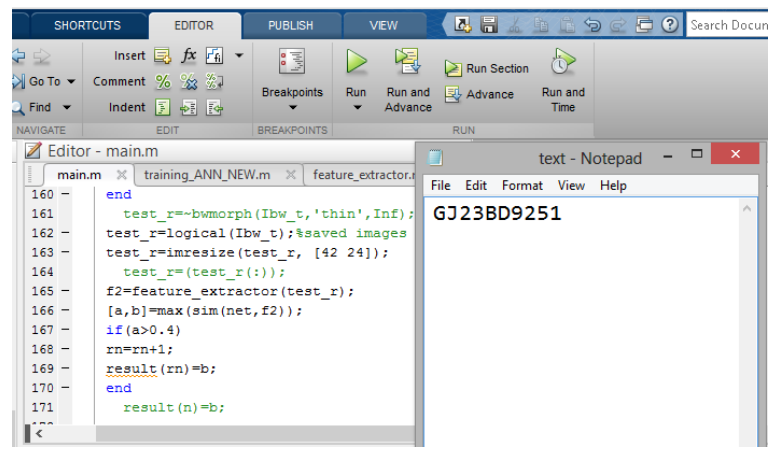

Figure 10. License Plate Recognition

Result of implementation of ALPR system on another case presented in following section. The subsequent images are as per the process flow.

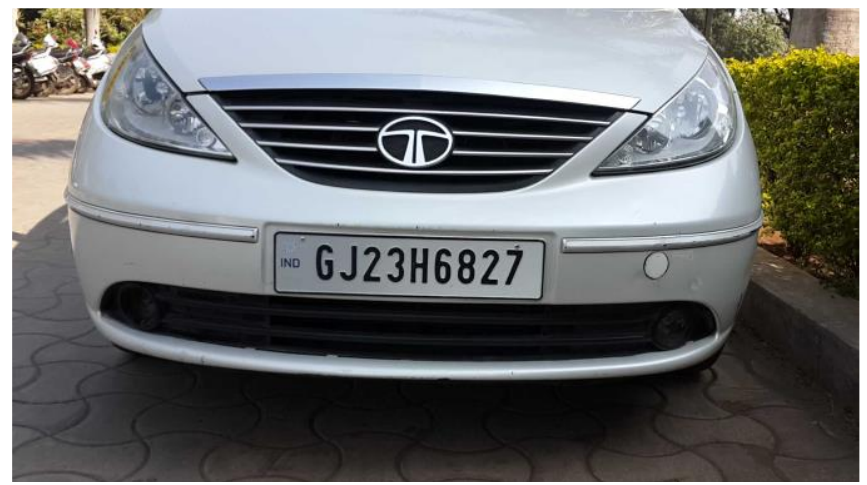

Figure 11. Input Image

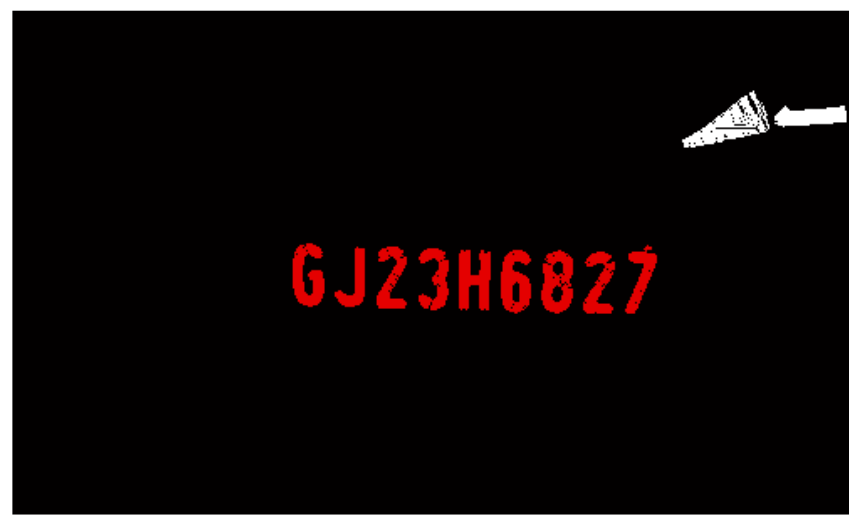

Figure 12. License Plate Detection

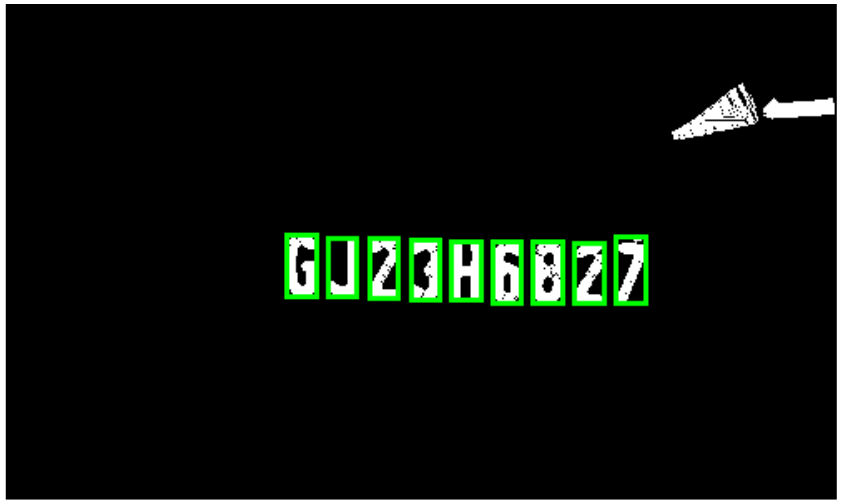

Figure 13. License Plate Segmentation

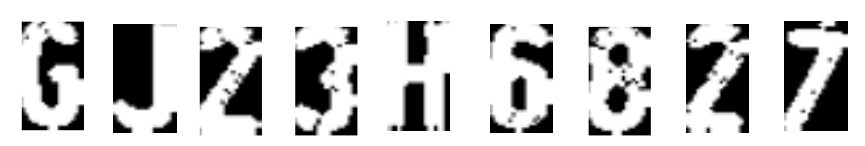

Figure 14. Segmented Characters

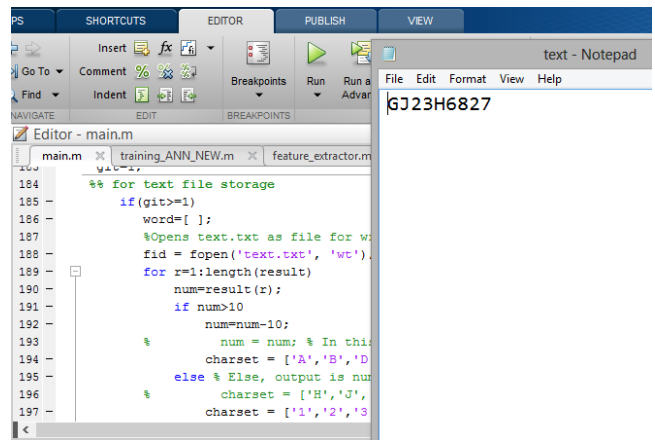

Figure 15. License Plate Recognition

\section{Performance Analysis}

Template matching based recognition has been implemented but it is not sufficient to recognize words from LP. Many nearer matched characters like, (5-S), (G-Q), (1-I) etc. can be miss recognized in different scenario. So to improve accuracy of the system and to avoid miss recognition ANN can be used. Time required to train ANN network is 15 minutes and 15 seconds. Figure 18 shows the structure of the network. Input data having total 86 node whereas hidden layer consist of 120 layers with logsig function for distribution. Output layer have total 17 classified classes by using tansig used during training. Once the network had trained with large dataset of around 300 characters, it is been tested and matched up-to to the higher accuracy level. The result of recognition can be seen in the Fig. 10 and 15. LP from the detected image had been successfully recognized. Once that completed, output was stored in excel file as well as text file. 

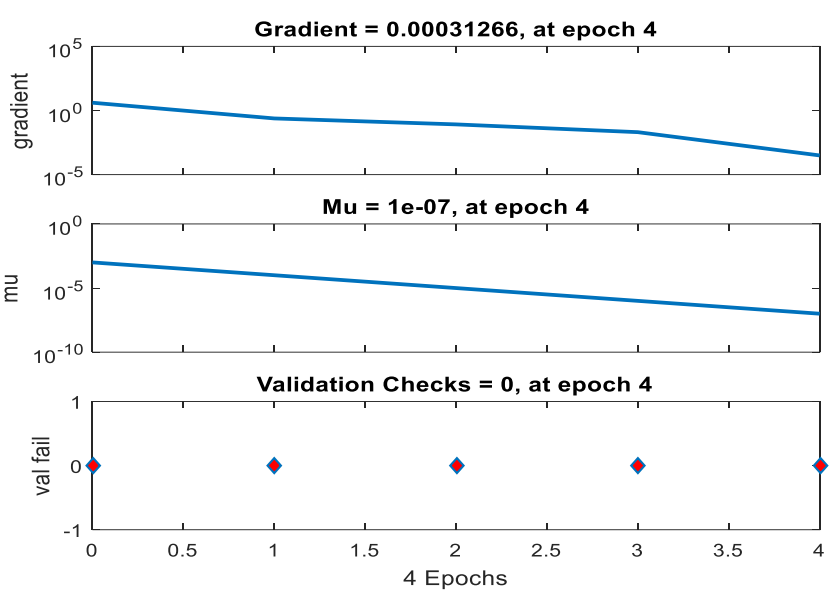

Figure 16. Training State

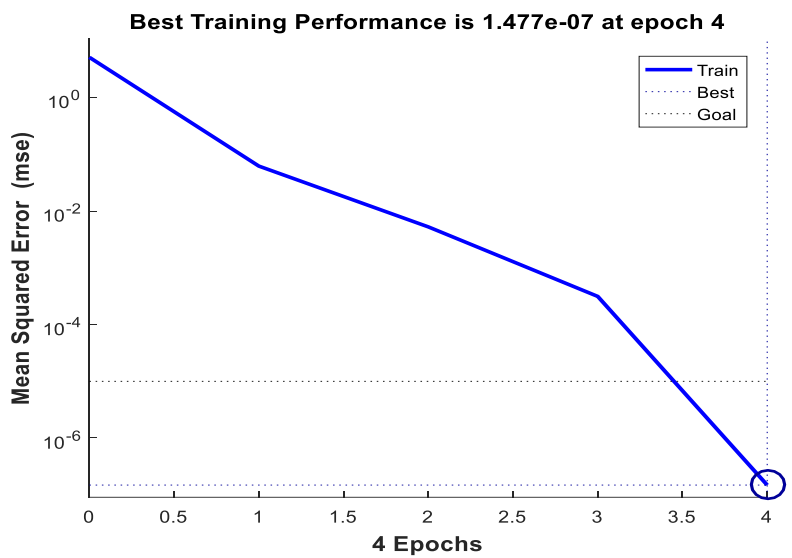

Figure 17. Performance

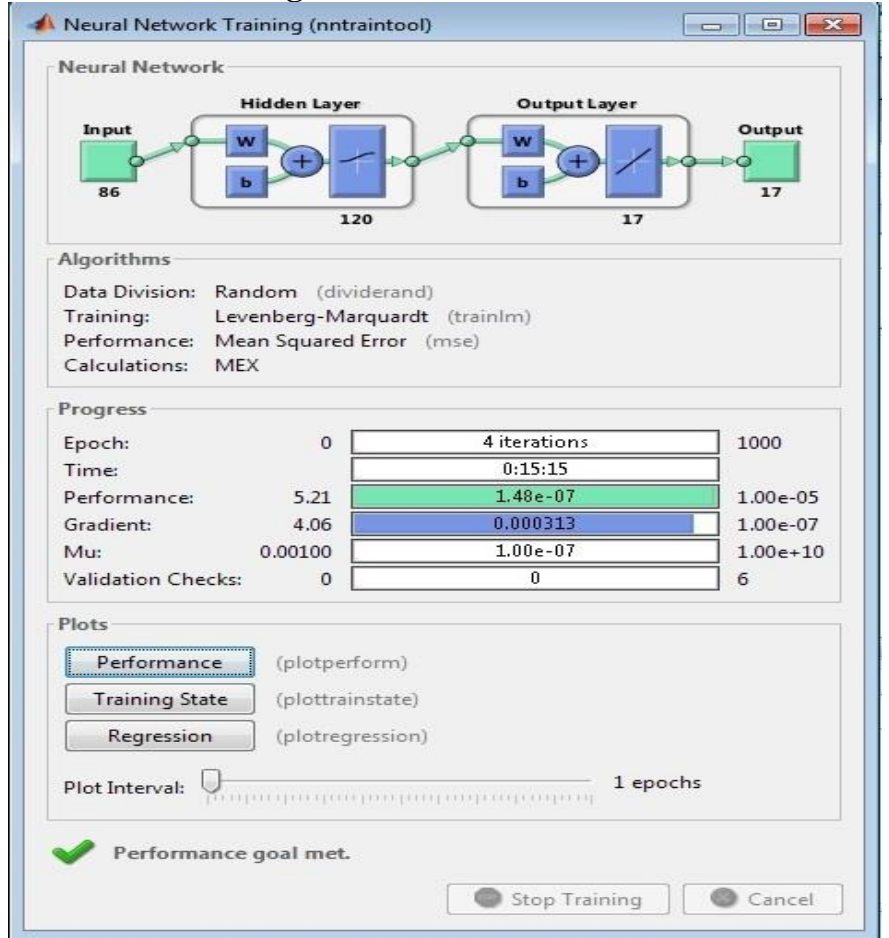

Figure 18. Structure of the network and Training procedure

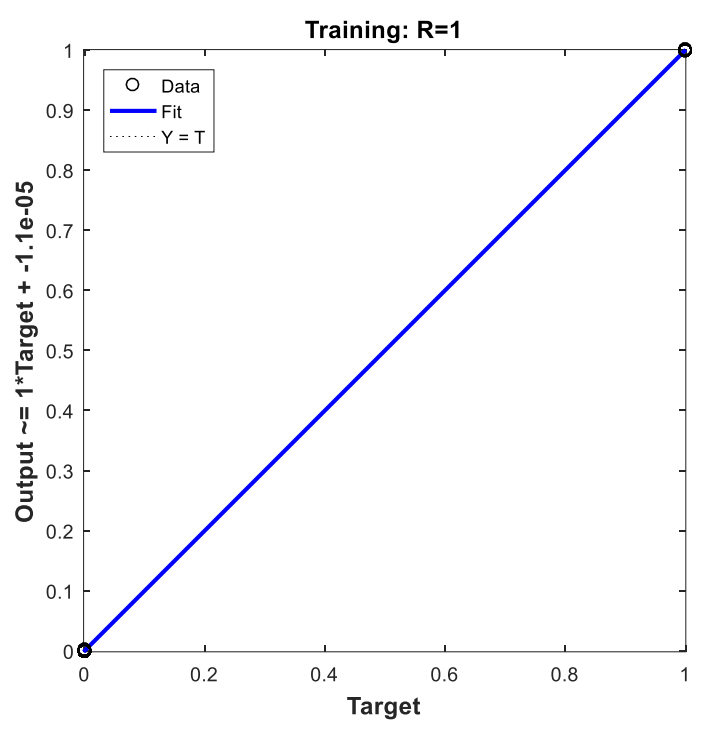

Figure 19. Regression

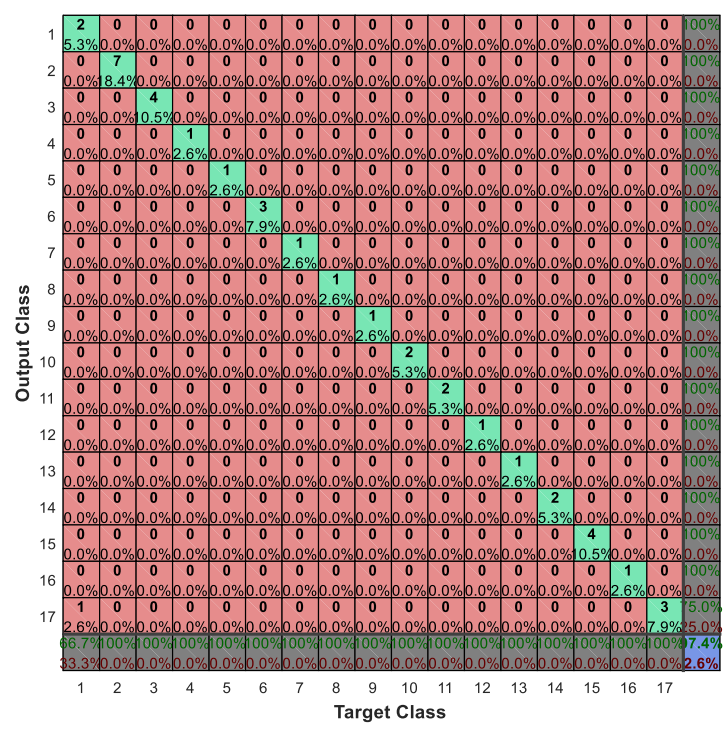

Figure 20. Confusion Matrix

\section{Conclusion}

With the help of SWT based LP detection method gives effective localization of License plates for different kind of images. An integrated approach give better segmentation result in dynamic condition. An algorithm for Automatic License Plate Recognition system has been developed for college premises successfully for live streaming data. Vehicles are detected effectively and Data-Log of license plate has been generated successfully by taking care of most of constraints at college premises. Compelling results are gotten for images having diverse difficulties for example, broken number plate, number plate in night mode, distinctive illumination conditions and motion variations so forth. The 
experimental results shows good accuracy compared to other methods suggested in literature.

\section{Acknowledgment}

Authors are grateful to GUJCOST for providing financial assistance through minor research project 2013-14 grant. Authors also grateful to Principal and Head, Electronics and Engineering division of the A. D. Patel Institute of Technology for continuous motivation, assistance and encouragement.

\section{References}

1. Surabhi Mohindra, Dr. Shailja Shukla, "License Plate Recognition (LPR) system for Indian Vehicle License Plate Extraction and Character Segmentation" International Journal Of Engineering Sciences \& Research Technology, Vol. 2 (7), pp. 1761-1765, July 2013.

2. S.Kranthi, K.Pranathi, A.Srisaila "Automatic Number Plate Recognition" International Journal of Advancements in Technology, Vol. 2, No 3, pp. 408 - 422, July 2011.

3. Abbas M. Al-Ghaili, Syamsiah Mashohor, Abdul Rahman Ramli, and Alyani Ismail, "Vertical-EdgeBased Car-License-Plate Detection Method", IEEE Transactions on vehicular technology, vol. 62, no. 1, pp. 26 - 38, January 2013.

4. D. Bradley and G. Roth, "Adaptive thresholding using the integral image", Journal of Graphics Tools, Vol. 12, no. 2, pp. 13-21, Jun 2007.

5. Xiehua Zhang, Shen Zhang, "A Robust License Plate Detection Algorithm Based on Multi-features", 2nd International Conference on Computer and Automation Engineering (ICCAE), Vol. 5, Singapore, pp. 598 -602, 26 - 28 February, 2010.

6. Nipa D. Modi, Chintan K. Modi, Chirag N. Paunwala, Suprava Patnaik, "Skew Correction for Vehicle License Plates using Principal Component of Harris Corner Feature", International Conference on Communication Systems and Network Technologies, pp. 339- 343, 2011.

7. Prathamesh Kulkarni, Ashish Khatri, Prateek Banga, Kushal Shah, "A Feature Based Approach for Localization of Indian Number Plates" IEEE International Conference on Electro/Information Technology, Windsor, pp. 157-162, 7 - 9 June, 2009.

8. Chirag N. Paunwala and Dr. Suprava Patnaiak, "License Plate extraction based on gradient and wavelet analysis", International Conference on Signal, System and Automation, pp. 41-44, 2009.

9. Chirag N. Paunwala, Dr. Suprava Patnaik, "Automatic License Plate Localization Using Intrinsic Rules Saliency", International Journal of Advanced Computer Science and Applications,
Special Issue on Image Processing and Analysis, pp. 105-111.

10. Chirag N. Paunwala, Suprava Patnaik," A Novel Multiple License Plate Extraction Technique for Complex Background in Indian Traffic Conditions", International Journal of Image Processing (IJIP) Vol. 4, Issue 2, pp. 106-118.

11. Boris Epshtein, Eyal Ofek, Yonatan Wexler, "Detecting Text in Natural Scenes with Stroke Width Transform", IEEE Conference on Computer Vision and Pattern Recognition (CVPR), San Francisco, CA, pp. 2963 - 2970, 13-18 June 2010.

12. Jung Jin Lee, "Adaboost for text detection in natural scene", International Conference on Document Analysis and Recognition, Beijing, pp. 429 - 434, 18 - 21st September.

13. S. Kim, D. Kim, Y. Ryu, and G. Kim, "A robust license-plate extraction method under complex image conditions," 16th International Conference on Pattern Recognition, Quebec City, QC, Canada, pp. 216-219, 2002.

14. Takashi Naito, Toshihiko Tsukada, Keiichi Yamada, Kazuhiro Kozuka, and Shin Yamamoto, "Robust License-Plate Recognition Method for Passing Vehicles under Outside Environment", IEEE Transactions on Vehicular Technology, Vol. 49, No. 6, pp. 2309-2319, November 2000.

15. K. V. Jobin, C. V. Jiji, P. R. Anurenjan, "Automatic number plate recognition system using modified stroke width transform", Fourth National Conference on Computer Vision, Pattern Recognition, Image Processing and Graphics (NCVPRIPG), 2013,

16. C. Patel, D. Shah, and A. Patel, "Automatic Number Plate Recognition System (ANPR): A Survey," International Journal of Computer Applications, vol. 69, pp. 21-33, may 2013.

17. J. Jagannathan, A. Sherajdheen, R. Muthu, and N. Krishnan, "License Plate Character Segmentation Using Horizontal And Vertical Projection with Dynamic Thresholding," IEEE International Conference on Emerging Trends in Computing, Communication and Nanotechnology, no. Iceccn, pp. 700-705, 2013.

18. Y. Yoon, K.-d. Ban, H. Yoon, and J. Kim, "Blob Detection and Filtering for Character Segmentation of License Plates," IEEE 14th International Workshop on Multimedia Signal Processing (MMSP), pp. 349-353, 2012.

19. M. K. Sarker and M. Kyou Song, "A Novel License Plate Character Segmentation Method for Different Types of Vehicle License Plates," IEEE International Conference on Information and Communication Technology Convergence (ICTC), pp. 84-88, 2014. 
20. R. Hitesh and S. Tanmoy, "An Automated Vehicle License Plate Recognition System," IEEE Computer Society, no. 1, 2015.

21. C.-H. Chen, T.-Y. Chen, M.-T. Wu, T.-T. Tang, and W.-C. Hu, "License Plate Recognition for Moving Vehicles Using a MovingCamera," IEEE 9th International Conference on Intelligent Information
Hiding and Multimedia Signal Processing, pp. 497500, Oct 2013.

22. H.Erdinc Kocer, K.Kursat Cevik, "Artificial neural networks based vehicle license plate recognition", Procedia Computer Science 3 (2011), Elsevier, pp. 1033-1037.

\section{Biographical notes}

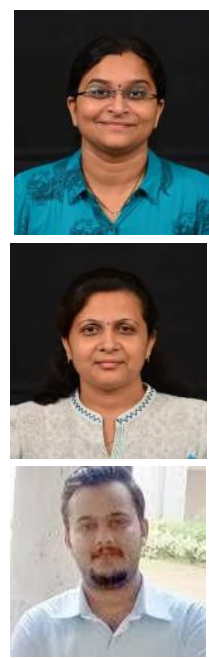

Dr. Heena Kher has received Ph.D. from Sardar Patel University, Vallabh Vidyanagar in 2014 and M.E. in Microprocessor System and Applications from M.S. University, Baroda in 2006. She is Assistant Professor in Electronics and Communication Engineering of A. D. Patel Institute of Technology, New Vallabh Vidyanagar, Gujarat, India. Her research interest includes Signal and Image Processing and Microcontrollers.

Dr. Hetal Patel has received Ph.D. from Sardar Patel University, Vallabh Vidyanagar in 2013 and M.E. in Microprocessor System and Applications from M.S. University, Baroda in 2005. She is Professor and Head in Electronics and Communication Engineering of A. D. Patel Institute of Technology, New Vallabh Vidyanagar, Gujarat, India. Her research interest includes Signal and Image Processing, Statistical signal processing, Microprocessors and Microcontrollers.

Tejendra Panchal has received M.E. in Signal processing and Communication and B.E. in Electronics and Communication from A D. Patel Institute of Technology, New V. V. Nagar in 2016 and 2013 respectively. His research interest includes digital image processing, Embedded systems and machine learning. 